Technical Note

\title{
Regional Urban Extent Extraction Using Multi-Sensor Data and One-Class Classification
}

\author{
Xiya Zhang, Peijun Li * and Cai Cai \\ Institute of Remote Sensing and GIS, School of Earth and Space Sciences, Peking University, \\ Beijing 100871, China; E-Mails: xyzhang58@gmail.com (X.Z.); caicai361448336@gmail.com (C.C.) \\ * Author to whom correspondence should be addressed; E-Mail: pjli@pku.edu.cn; \\ Tel./Fax: +86-10-6275-7364.
}

Academic Editors: Ioannis Gitas and Prasad S. Thenkabail

Received: 9 February 2015 / Accepted: 29 May 2015 / Published: 9 June 2015

\begin{abstract}
Stable night-time light data from the Defense Meteorological Satellite Program (DMSP) Operational Line-scan System (OLS) provide a unique proxy for anthropogenic development. This paper presents a regional urban extent extraction method using a one-class classifier and combinations of DMSP/OLS stable night-time light (NTL) data, MODIS normalized difference vegetation index (NDVI) data, and land surface temperature (LST) data. We first analyzed how well MODIS NDVI and LST data quantify the properties of urban areas. Considering that urban area is the only class of interest, we applied the one-class support vector machine (OCSVM) to classify different combinations of the three datasets. We evaluated the effectiveness of the proposed method and compared with the locally optimized threshold method in regional urban extent mapping in China. The experimental results demonstrate that DMSP/OLS NTL data, MODIS NDVI and LST data provide different but complementary information sources to quantify the urban extent at a regional scale. The results also indicate that the OCSVM classification of the combination of all three datasets generally outperformed the locally optimized threshold method. The proposed method effectively and efficiently extracted the urban extent at a regional scale, and is applicable to other study areas.
\end{abstract}

Keywords: urban extent; OCSVM; DMSP/OLS; NDVI; land surface temperature; one-class classification 


\section{Introduction}

Urban areas occupy a small fraction of the earth's surface, but they significantly impact ecosystems and climates at local, regional, and global scales. This is because of land cover conversion, the release of anthropogenic greenhouse gases, and loss of biodiversity [1-8]. As such, accurate and timely information about the extent and spatial distribution of urban areas (especially at regional and global scales) is crucial and significant for a diverse range of applications. These applications include land use change [9], energy consumption [10], and global climate change [5].

High-resolution images provide detailed spatial information for mapping individual cities [11], but they are not sufficient for examining urban patterns at a large scale [12]. Although moderate resolution images have been used for land cover mapping at continental scales [13-15], the time and labor required for processing and interpreting these images make it difficult to map urban areas at a large scale. Common cloud conditions over a large area also make it difficult to collect a large number of good-quality images within the same year [16]. Satellite data at spatial resolutions of 100-1000 m provide reliable data regarding the urban extent and spatial distribution at a large scale, because of their synoptic view and wide coverage. Many researchers have investigated mapping urban areas at regional and global scales from coarse resolution data over the past two decades [16-22].

Different coarse resolution data have been used in urban extent mapping, for example, Terra Moderate Resolution Imaging Spectroradiometer (MODIS) data [21], Medium Resolution Imaging Spectrometer (MERIS) data [23], and Defense Meteorological Satellite Program/Operational Line-scan System (DMSP/OLS) nighttime light (NTL) data [24]. The DMSP/OLS NTL data are regarded as the most promising among these data sources [25], because they have the unique capability of recording human activities on the earth's surface by observing artificial lighting at night [17,24], and have a relatively long historical archive. Thus, DMSP/OLS NTL data can be used to accurately, economically, and unambiguously map the urban extent at a regional scale [24-26].

Although the DMSP/OLS NTL data are valuable for regional and global urban mapping and for analyzing human activities [17,25-31], the detected lit areas are substantially larger than the ground truth. This is called the "blooming" effect [8,24], and is the result of scattered light in the atmosphere and the photomultiplier's response to bright emission sources [8,32]. Various methods have been developed to map urban areas using DMSP/OLS NTL data [16,24,26,29,33-38]. Global fixed thresholding and locally optimized thresholding methods are the mostly common and efficient techniques [16,24,29,35]. Although existing studies have demonstrated the potential of thresholding techniques in reducing blooming, there are some problems [8,16,24,29,31,35]. A major challenge related to the global fixed thresholding method is how to determine a global threshold value for all urban areas, because of significant regional variations in environmental and socioeconomic development levels [8,24,29]. The global fixed thresholding method may overestimate urban areas with high development levels, and underestimate or not identify small urban areas with low development levels [8,29]. Although the locally optimized threshold is based on the development level of each urban area and is relatively accurate, there are currently no general guidelines for selecting locally optimized threshold values. Moreover, selecting locally optimized thresholds is laborious and time consuming when mapping urban areas at regional or global scales $[16,35,36]$. 
Considering that the vegetation index is negatively correlated with urban features, Lu et al. [16] proposed an approach that jointly used DMSP/OLS NTL data, MODIS normalized difference vegetation index (NDVI) data, and Landsat TM/ETM+ data to map fractional settlements through regression models and overcome problems in the threshold selection. Their results showed that a combination of DMSP/OLS NTL and MODIS NDVI data was better than the use of DMSP/OLS NTL data alone. In another study, a method based on a support vector machine (SVM) was proposed to classify a combination of DMSP/OLS NTL and SPOT VEGETATION NDVI data to extract urban areas [35]. The SVM-based method achieved comparable results to the locally optimized threshold method, but avoided the tedious trial-and-error procedure for determining a local threshold [35]. Pandey et al. [36] and Yang et al. [37] also used DMSP/OLS NTL and SPOT VEGETATION NDVI data with modified SVM-based methods to map the urban extent in Indian and China, respectively. All these studies indicated the combination of DMSP/OLS and NDVI data is promising when mapping urban areas at a regional scale.

However, these existing studies have two problems. First, they only incorporated NDVI data to improve the performance of the urban extent mapping based on DMSP OLS NTL data. Related studies found that other surface properties, e.g., land surface temperature (LST), are also closely related to urban features. For example, the local LST is influenced by urbanization [39-42]. Urban areas typically display higher LST values compared with surrounding rural areas, which is related to the urban heat island effect $[41,42]$. Thus, the LST data from Landsat TM/ETM+ have been used to estimate impervious surfaces [43]. The LST data were also combined with NDVI data for land cover mapping at regional scale [44-47]. Lambin and Ehrlich showed that a combination of LST and NDVI data achieved more accurate land cover classifications at a continental scale, when compared to the use of NDVI alone [48,49]. These studies indicated that LST and vegetation indices are useful in discrimination between different land cover classes. However, LST data were rarely used to extract urban areas at regional or global scales [50]. Considering the capability of LST and NDVI data for discrimination between land cover classes, and the complementary characteristics of DMSP/OLS NTL, NDVI, and LST data in separating urban areas from non-urban areas, combination of these three data sets may provide new insights for mapping urban areas at regional scale.

Second, existing methods based on SVM classification assumed that the mapping was a two-class classification problem (i.e., urban and non-urban) [35-37]. However, mapping urban extent at regional or global scales is essentially an unbalanced classification task, because urban areas typically occupy a much smaller area than non-urban areas. Moreover, the non-urban areas show much significant variability in multi-sensor data values, e.g., high NTL data values and low NDVI and LST values (illuminated marine vessels), low NTL data values and different NDVI values (soil and vegetation areas), and thus are difficult or very expensive to sample. Furthermore, urban areas are the only class of interest in mapping urban area at regional scale in this paper. Thus, new methods, which can effectively extract urban areas from complex backgrounds with significant variability (non-urban areas), but do not need to collect samples from complex backgrounds (non-urban areas), are required. In this context, the problem of extracting urban extent from multi-sensor data possesses the features of the one-class classification problem, where only data from the target class (i.e., urban area in this study) are available and well sampled [51,52]. One-class classification methods have successfully been used in extracting specific land cover types and change types [53-56]. Among all one class classification methods adopted 
in the remote sensing field, one-class support vector machine (OCSVM) [54-56] and support vector data description (SVDD) [53] have been widely discussed.

Considering the problems mentioned above, the purpose of this study is to evaluate the use of combination of NTL, NDVI, and LST data, and a one-class classification method for regional urban mapping. The specific objectives are: (1) to evaluate the feasibility and effectiveness of including LST and NDVI data to describe urban areas; (2) to develop and evaluate a regional urban extent extraction method using the OCSVM, a recently developed one-class classifier, and multi-sensor data, i.e., combination of DMSP/OLS NTL, MODIS NDVI, and LST data. The paper is organized as follows. Section 2 describes study area and data. The regional urban mapping method using multi-sensor data and OCSVM method is presented in Section 3. Experimental results are given in Section 4, while discussion of the results and findings are in Section 5. Finally, conclusions are drawn in Section 6.

\section{Study Area and Data}

China has been experiencing rapid and large-scale urbanization since the opening and reform policy was established in the late 1970s [57,58]. By the end of 2012, the urban population of China was 52.6\% of the total population (712 million), rising from $26 \%$ in 1990 [59,60]. We selected China as our study area, because of the scale of its urban expansion and the uneven development pattern.

Urbanization levels in China vary greatly across the country [61,62]. The eastern coastal region experienced significantly more urbanization, followed by the central region, and finally by the west. The differences in urbanization levels across China are attributed to the heterogeneous socioeconomic development. The eastern coastal region covers the most developed areas of China, where the majority of the population lives in urbanized areas. The economy in this area is undergoing a transition to a mixture of manufacturing and service industries. In the central region, the economy relies more on manufacturing, and there is less urbanization. The western region includes the vast under-developed provinces. In this area, agriculture and manufacturing are the main economic activities, and there is the least urbanization [61]. There are also large differences in the urban population distributions of different parts of China. In 2008, more than half the cities and urban population of China were distributed on the east coast, whereas $19 \%$ of cities and $17.7 \%$ the urban population were distributed in the west [62]. These different urbanization densities and patterns provide ideal experimental regions for evaluating the applicability of the proposed methods.

In this paper, we used a combination of DMSP/OLS stable NTL data, Terra MODIS NDVI data, and LST data to extract urban areas at a regional scale. The DMSP/OLS NTL data measure light on the earth's surface caused by human settlements, gas flares, fires, and shipping fleets. The DMSP/OLS stable NTL data are annual cloud-free composites based on the highest-quality data and a number of constraints [28]. The value at each pixel is a digital number (DN) from 0-63 with a $1 \mathrm{~km}$ spatial resolution, spanning longitudes of $-180^{\circ}-180^{\circ}$ and latitudes of $-65^{\circ}-75^{\circ}$. The Terra MODIS NDVI data (MOD13A2) was provided every 16 days at a $1 \mathrm{~km}$ spatial resolution. They were computed from atmospherically corrected bidirectional surface reflectances that have been masked for water, clouds, heavy aerosols, and cloud shadows [63,64]. The MODIS LST data used in this study are MOD11A2 daytime LST data. The MODIS global LST data (MOD11A2) were derived from the daily $1 \mathrm{~km} \mathrm{LST}$ 
product (MOD11A1), and the average values of clear-sky LSTs during an eight day period were stored on a $1 \mathrm{~km}$ sinusoidal grid [65].

All three data sets were acquired in 2006. The NDVI data used in this study were acquired from April to October 2006, because this is the growing season. The LST data were collected from January to December 2006. The three datasets were re-projected using Albers equal-area conic projection and then clipped according to Chinese administrative boundaries (Figure 1). We applied a maximum value composition [66] to all the NDVI images to create a yearly maximal NDVI image (Figure 1b). Yearly maximal NDVI can effectively reduce the impact of bare soil [16]. We also created a yearly mean LST image (Figure 1c) using all the daytime LST images, because mean LST is insensitive to seasonal variations and has been extensively used in studies of urban heat islands [67,68].

Additionally, we used 50 Landsat TM/ETM+ images covering 55 urban areas to validate the urban extent mapping results using multi-sensor coarse resolution data. Landsat TM/ETM+ images with a $28.5 \mathrm{~m}$ spatial resolution were acquired for 2005-2007. Three quarters of these images were acquired for 2006, and the others were from 2005 or 2007 because there were no suitable images available for 2006. All the selected Landsat TM/ETM+ images with the universal transverse mercator coordinate system were re-projected using Albers equal-area conic projection.

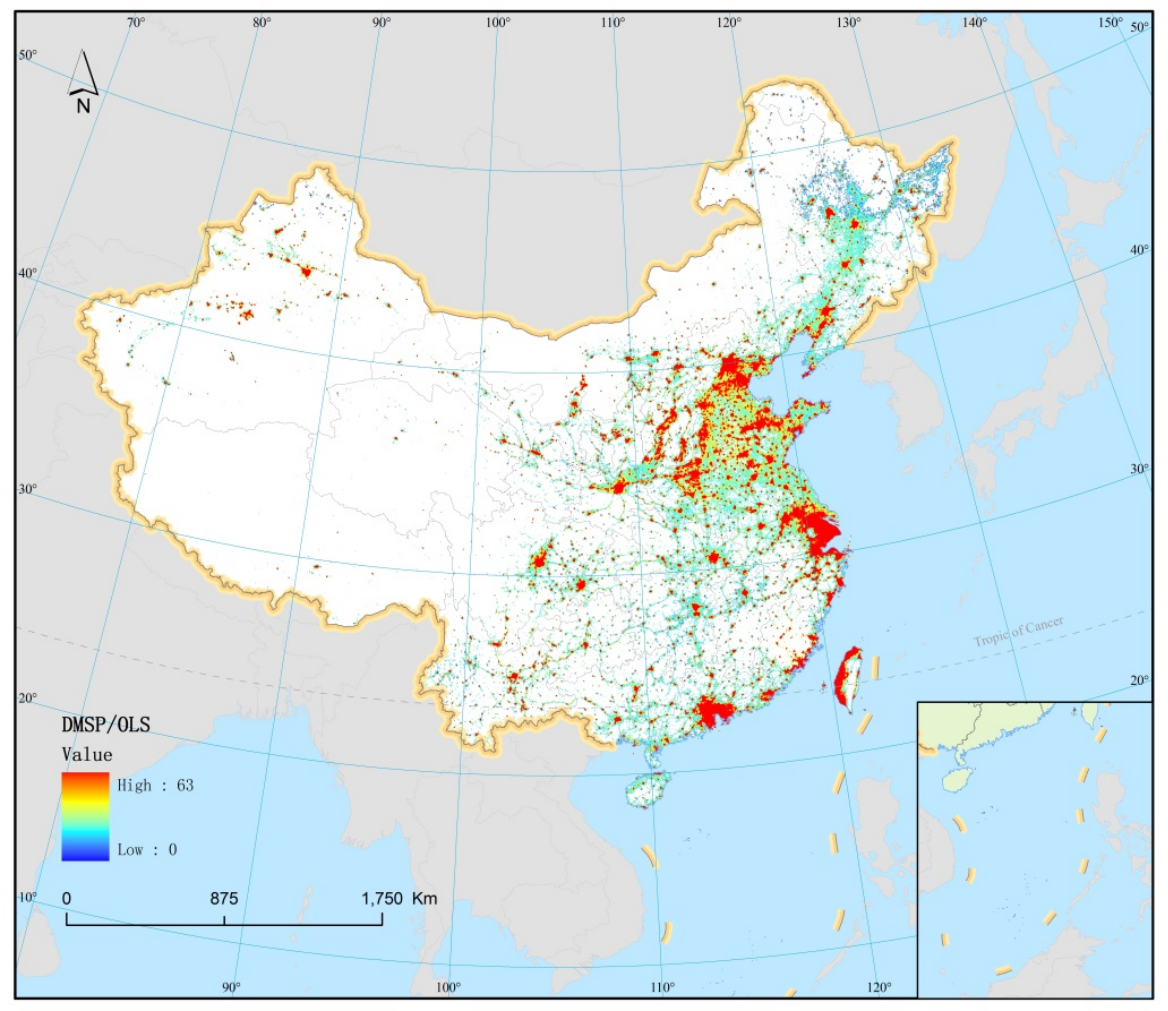

(a)

Figure 1. Cont. 


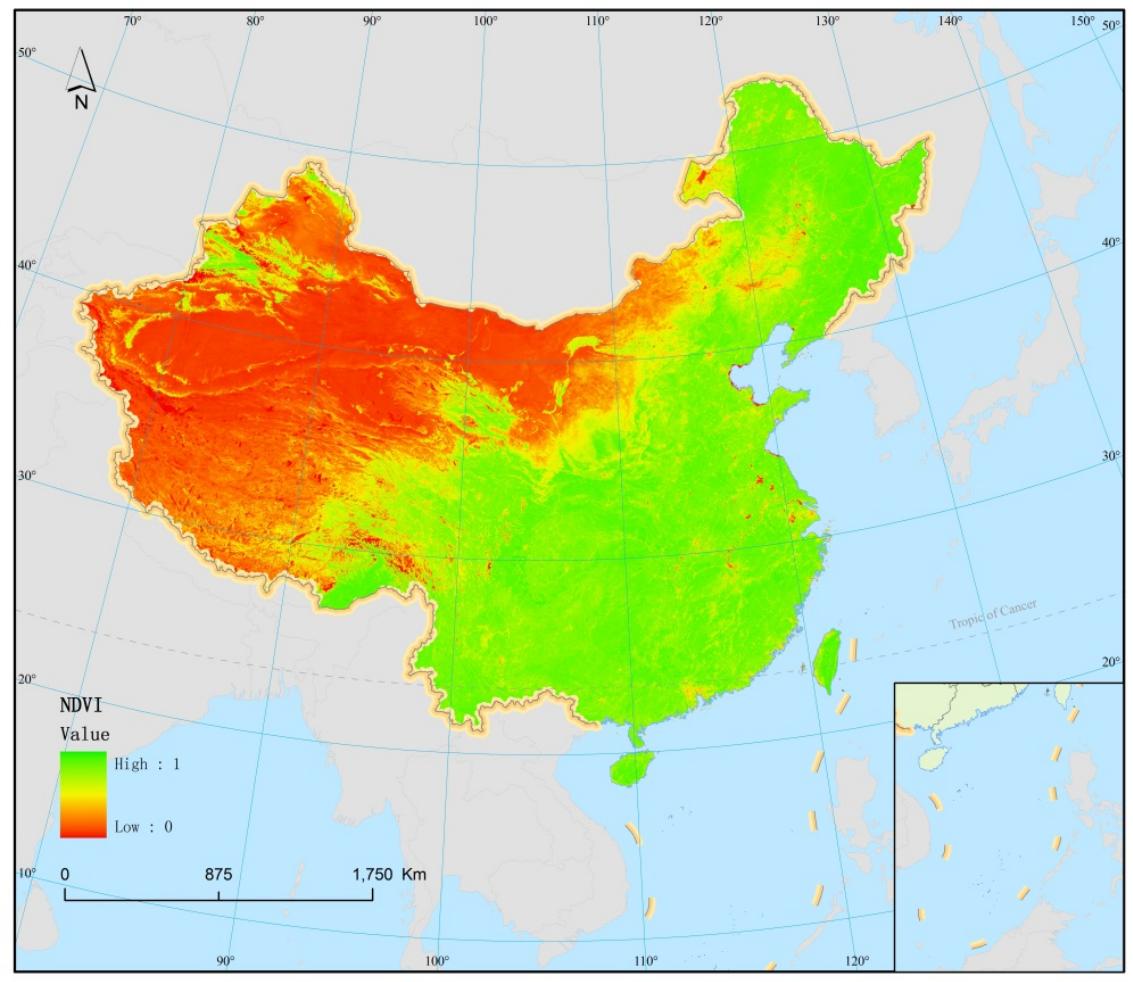

(b)

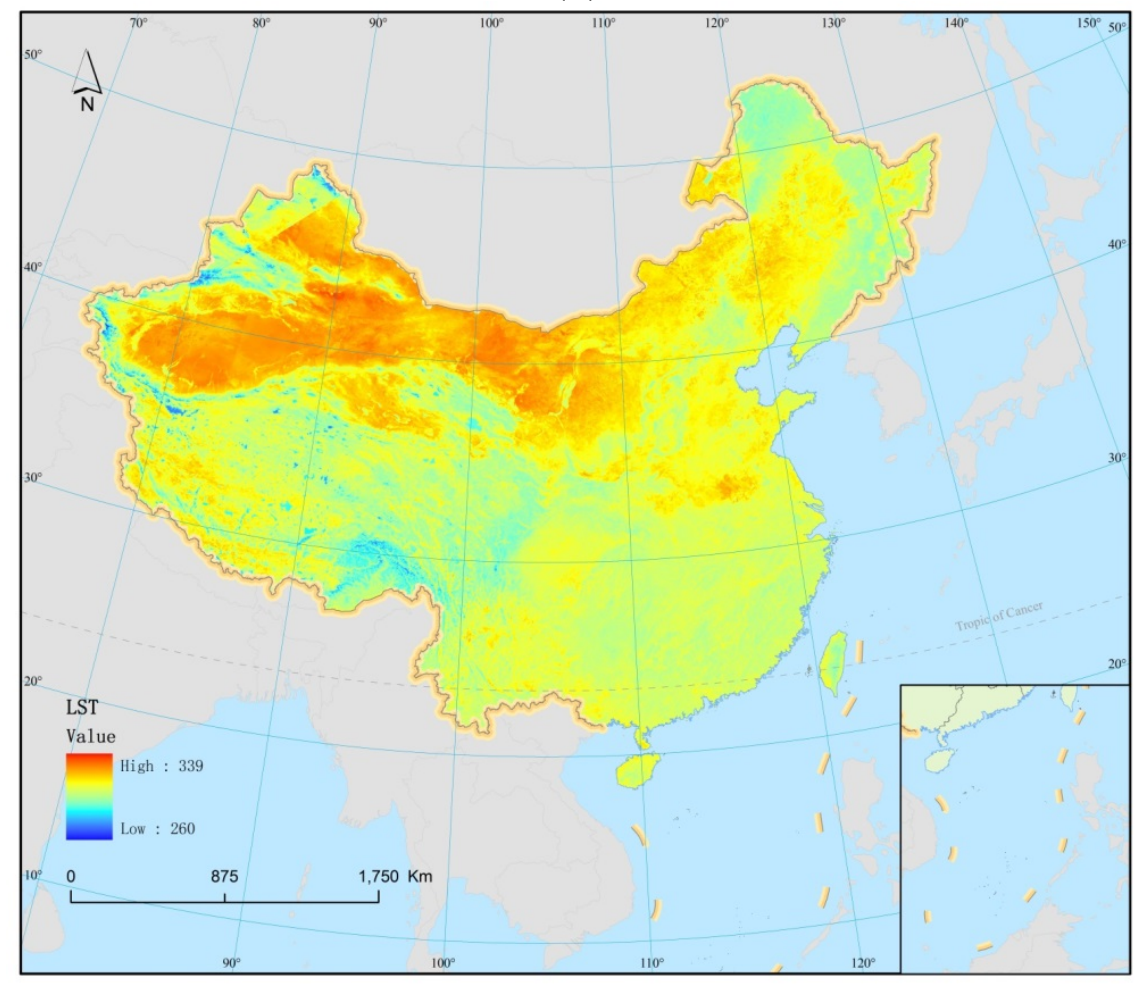

(c)

Figure 1. (a) DMSP/OLS stable NTL data of China in 2006; (b) Terra MODIS NDVI maximum value composition of China in 2006; (c) Terra MODIS LST yearly mean image of China in 2006. The DMSP/OLS NTL data are digital values of lighted pixels. The white background pixels, recorded as zero in DMSP/OLS NTL data, were considered non-urban land. The unit of LST is Kelvin temperature. 
The 55 selected cities with different development levels and urban populations are shown in Figure 2. Urbanization levels in the selected cities vary greatly, and these cities are also representative cities from each province of China. Specifically, they include cities from coastal regions (such as the Pearl River Delta, the Yangtze River Delta, and the Beijing-Tianjin-Tangshan region), which are called the 'three engines of China's economy', and cities from inland and under-developed provinces in the west. Variations in the urban population among the selected cities are obvious (Figure 2). For example, the populations of these 55 cities varied from less than 220,000 (Lhasa) to over 10 million (Shanghai) in 2006 [69]. For the sake of comparison, the selected cities were divided into three groups according to their urban populations. The first group included cities with urban populations greater than 2 million (big cities). The second group included cities with urban populations greater than 1 million and less than 2 million (medium cities). The third group included cities with urban populations less than 1 million (small cities).

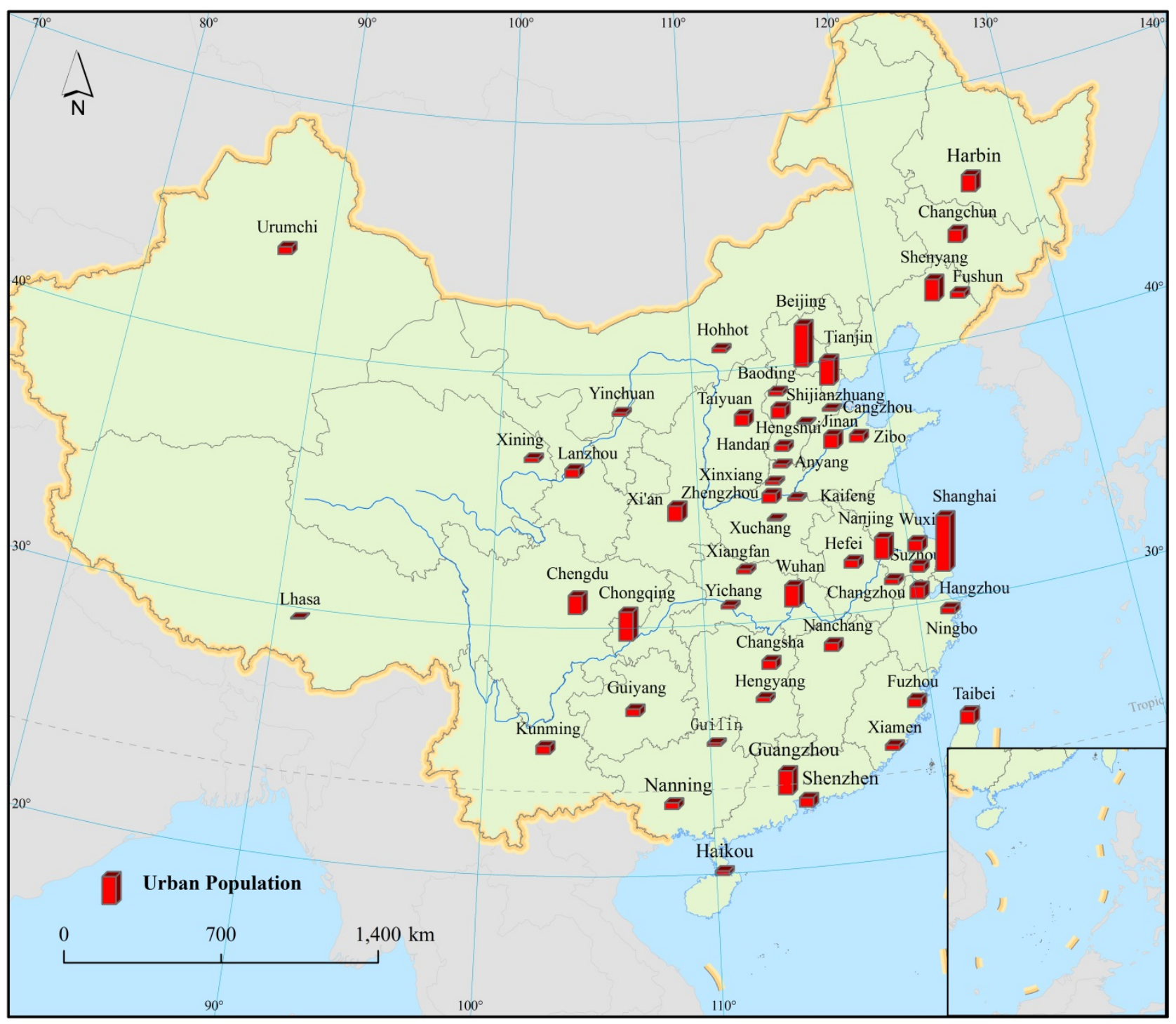

Figure 2. Chinese cities selected for result validation and their urban populations in 2006 [69].

We also obtained the socioeconomic statistics for these cities in 2006 from the Chinese Statistical Yearbook, 2007 [69]. Statistical data for urban built-up areas were also used for reference purposes. 


\section{Methods}

In this paper, we propose a method for extracting regional urban extent based on the one-class SVM (OCSVM) classification method and a combination of DMSP/OLS NTL, MODIS NDVI, and LST data. We first analyzed three datasets to fully understand the proposed method and to evaluate the effectiveness of MODIS NDVI and LST data when applied to regional urban extraction. We then applied the OCSVM method and different data combinations in regional urban extraction. The urban extent extracted using different methods was finally evaluated using the reference data.

\subsection{Data Analysis}

Urban areas are typically locations with high densities of buildings and low levels of vegetation. Urban areas also generally experience warmer temperatures than their rural surroundings because of the "urban heat island" effect, which is one consequence of the concentration of buildings and lack of vegetation in urban cores [70,71]. Gallo et al. [39] compared AVHRR NDVI data and DMSP/OLS NTL data using DN profiles across a latitudinal transect to examine the relationships between NDVI and NTL data in urban and rural areas. Zhang et al. [70] examined the relationship between DMSP/OLS NTL and MODIS NDVI data using DN profiles. They found that NDVI values in urban regions are relatively low, whereas DMSP/OLS NTL values gradually increase towards the urban core along the transect [39,70]. Temperature differences between urban cores and their surrounding rural regions has been extensively investigated and documented [42,71-73].

We used profiles from selected urban areas to demonstrate the relationships among these three datasets, as in previous studies $[39,70]$. Given that the three data sets have very different data ranges, and the yearly maximal NDVI image ranges between 0 and 1, we normalized the DMSP/OLS NTL and MODIS LST so that they ranged between 0 and 1. Specifically, the DMSP/OLS NTL data were normalized using the following equation:

$$
N T L_{\text {nor }}=\frac{N T L-N T L_{\min }}{N T L_{\max }-N T L_{\min }}
$$

where $N T L_{\text {nor }}$ is the normalized value of the DMSP/OLS NTL image, $N T L$ denotes the original DMSP/OLS NTL value of a pixel. $N T L_{\max }$ and $N T L_{\min }$ are the minimum and maximum values of the DMSP/OLS NTL image, i.e., 0 and 63. The MODIS LST data were normalized using the following equation:

$$
L S T_{\mathrm{nor}}=\frac{L S T-L S T_{\min }}{L S T_{\max }-L S T_{\min }}
$$

where $L S T_{\text {nor }}$ is the normalized MODIS LST value and $L S T$ is the original LST value of a pixel. $L S T_{\max }$ and $L S T_{\min }$ are the maximum and minimum values of the original MODIS LST image.

Three cities of different sizes (in population) were selected for comparison: Beijing (representing big cities with urban populations over 2 million), Suzhou of Jiangsu Province (representing medium cities with urban populations between 1 and 2 million), and Xuchang of Henan Province (representing small cities with urban populations less than 1 million). The normalized DN profiles of the DMSP/OLS NTL, MODIS NDVI, and LST data across a latitudinal transect are shown in Figure 3. The DMSP/OLS NTL values in Beijing were much more saturated in the urban core area with DN values of almost 63 . The LST 
values in Beijing were significantly higher than surrounding areas, whereas the NDVI values were lower than in the surrounding areas with a trough in the urban core. The LST and NDVI values fluctuated within the urban area (Figure 3a). For Suzhou, the DMSP/OLS NTL values were less saturated than those in Beijing. MODIS LST values peaked in the urban core, and NDVI values troughed. MODIS NDVI values varied within the urban area, whereas the LST values changed smoothly (Figure 3b). For Xuchang, the DMSP/OLS NTL values for the urban area were much lower than the other two cities. The NDVI decreased and the DMSP/OLS NTL values increased towards the urban areas. The DMSP/OLS NTL and LST values peaked in the urban core (Figure 3c).

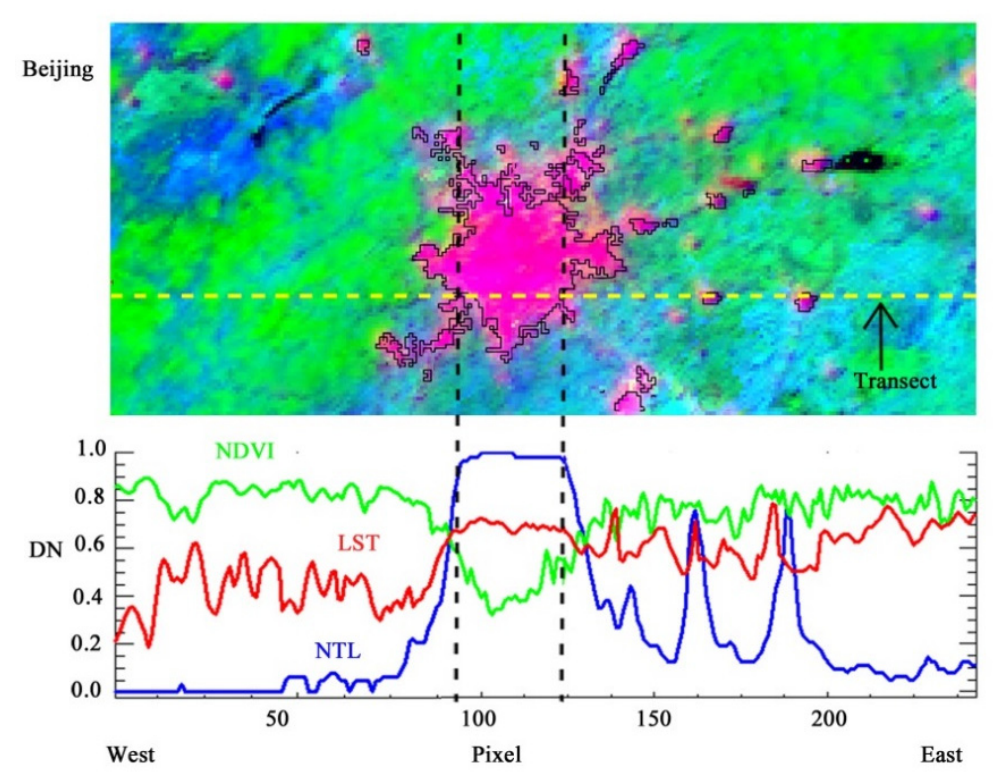

(a)

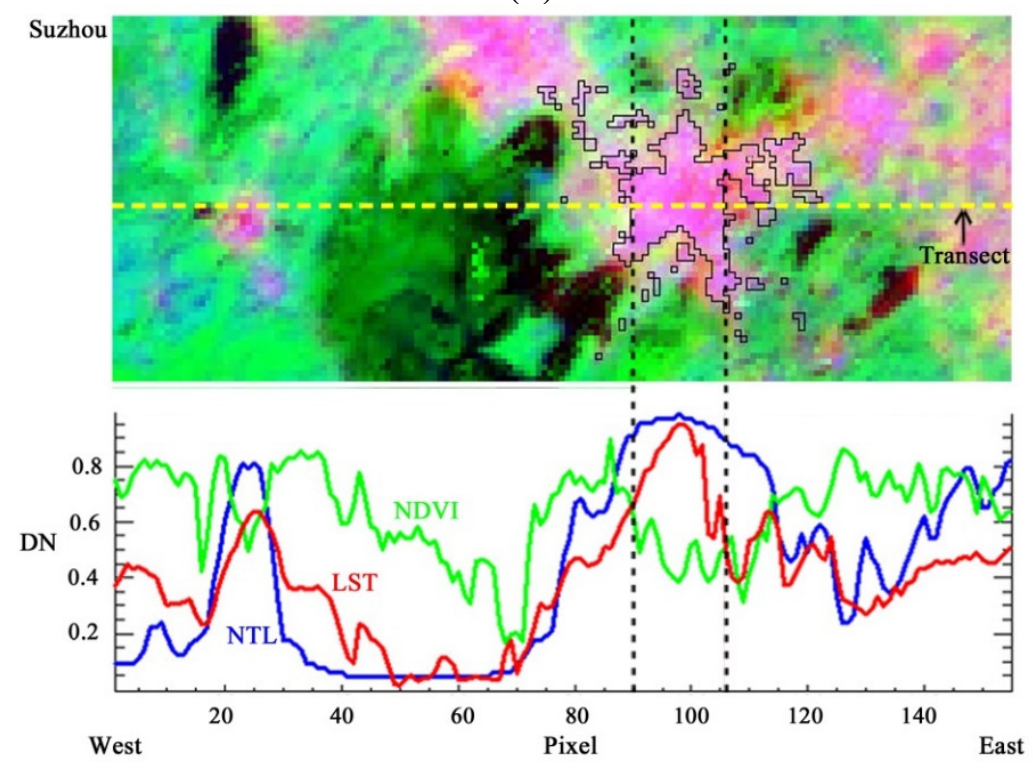

(b)

Figure 3. Cont. 


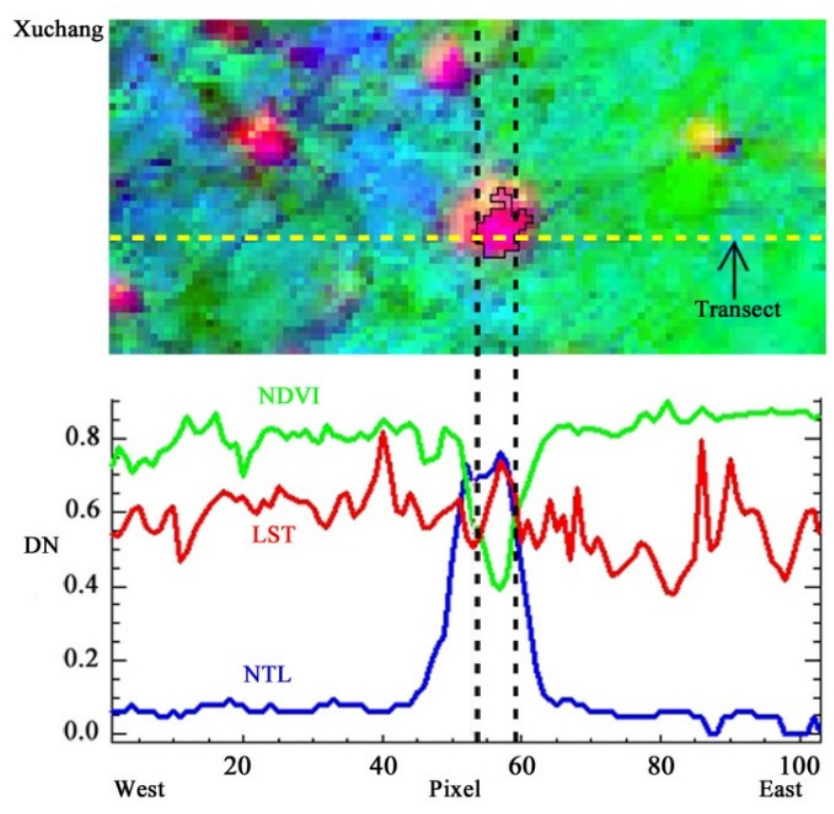

(c)

Figure 3. The color composite images (DMSP/OLS NTL, MODIS NDVI and LST as R, G and B) and their latitudinal transects of DMSP/OLS NTL (blue), MODIS NDVI (green), and MODIS LST (red) data for Beijing (a), Suzhou (b) and Xuchang (c).

It is evident from the transects of these three cities that urban areas typically display relatively high DMSP/OLS NTL and MODIS LST values, and low NDVI values. The opposite is true in rural regions (Figure 3). It is also very clear that the lit areas detected by DMSP/OLS NTL data are much larger than the ground truth extracted from Landsat TM images (Figure 3). Furthermore, the blooming effect is different for cities of different sizes. For example, the blooming effect in Beijing is more significant than that in Xuchang and Suzhou.

Based on the above data analysis, the MODIS LST and NDVI data provide information that is complementary to the DMSP/OLS data. Specifically, the MODIS NDVI and LST data provide information that can be used to extract the urban extent using vegetation and thermal signals. Thus, we expect that a combination of the three data sets will be useful.

Beijing has NTL values of more than 60, Suzhou has NTL values of less than 55, and Xuchang has NTL values of less than 50 (Figure 3). These different NTL values may represent different development levels. Therefore, it is inappropriate to use a global threshold value to extract these urban areas, because the thresholds used for different cities should be different. Considering this, we selected a locally optimized threshold method to validate the proposed methods.

\subsection{Extraction of Urban Areas Using Multiple Sensor Data}

From the analysis in the previous section, MODIS NDVI and LST data provide additional information for mapping the urban extent. Thus, a combination of the three datasets may improve the mapping results when compared with only using DMSP/OLS NTL data. To jointly use the three datasets, we need an appropriate supervised classifier for the multi-sensor data. Although two-class classifiers were used to classify multi-sensor data and obtained promising results [35-37], urban area is the only class of interest 
(or the target class) and only occupies a small area when compared to non-urban areas. Moreover, non-urban areas include diverse land cover classes, showing significant variability in multi-sensor data values, as described in the Introduction. Thus, mapping urban areas is essentially an unbalanced classification problem. Considering this, we used a one-class classification method [52] instead of traditional two-class classifiers. One-class classification methods have been successfully used to extract a specific land cover class and detect related changes $[53,55,56]$. Only samples from the target class are used during the training process, and no information about the counterpart (outlier class) is required. The boundary between the two classes has to be estimated from data of the only available target class. The task is to define a boundary around the target class, such that it encircles as many target examples as possible and minimizes the chance of accepting outliers [52].

OCSVM [51] is one of the most widely used one-class classification methods in remote sensing, and was selected as the classifier in this study. The OCSVM may be viewed as a special two-class SVM classifier, where all the training data lie in the first class (target class) and the origin is the only member of the second class (outlier class). The OCSVM algorithm first maps input data into a high-dimensional feature space via a kernel function, and then iteratively finds the maximal margin hyperplane that best separates the training data from the origin [51]. Using kernel functions, solving the OCSVM optimization problem is equivalent to solving the dual quadratic programming problem. Using the kernel function to project input vectors into a feature space, nonlinear decision boundaries are allowed. Generally, four types of kernels are often used: linear, polynomial, sigmoid, and Gaussian radial basis function (RBF) kernels. In this study, we use the RBF kernel, which has been commonly used for the OCSVM [51,52].

For urban extent mapping using multi-sensor data, rather than using training samples from two classes, we only use training data from the urban class with the OCSVM classifier. Additionally, instead of only using DMSP/OLS NTL and NDVI data when selecting the training samples in previous studies [35-37], we used a combination of all three datasets. We selected the urban training samples according to the statistical features of the DMSP/OLS, NTL and NDVI data for the 55 selected cities. We calculated the mean values for the three datasets in urban areas, and derived the urban extents from the Landsat TM/ETM+ data. Pixels that simultaneously satisfied three conditions were selected as candidate training samples: (1) DMSP/OLS NTL DN values greater than 30; (2) MODIS NDVI values greater than 0 and less than 0.4; and (3) MODIS LST values greater than $290 \mathrm{~K}$. Compared with only using DMSP/OLS NTL and NDVI data to select the training samples (e.g., [35]), using all three datasets removes the pixels containing gas flares, illuminated marine vessels, and water. This produces more reliable training samples. Because the study area is very large, this process generated a large number of training samples. We selected a subset of these as the final training set. Specifically, we randomly selected 8023 training samples (10\% of all reference samples) used in the OCSVM classification of multi-sensor data.

To evaluate the effectiveness of the different data sets for mapping urban extent, we used three data combinations: (1) DMSP/OLS NTL and MODIS NDVI data; (2) DMSP/OLS NTL and MODIS LST data; and (3) DMSP/OLS NTL, MODIS NDVI, and LST data.

\subsection{Validation}

To validate the effectiveness of the proposed methods and the different data combinations, we classified the Landsat TM/ETM+ data from 55 cities to extract urban areas. The obtained classification 
results were used as reference data. Because the Landsat ETM+ data has a much finer resolution than the DMSP/OLS NTL data, the classification results were sufficiently accurate to be used as the ground truth. Landsat TM/ETM+ data have been extensively used to validate urban area mapping results at regional scales using DMSP/OLS NTL data [8,29,35,37,74].

The SVM classifier [75] was used to classify Landsat TM/ETM+ images. Four land cover classes are generally recognized: urban area, vegetation (including forest, grass, and farmland), water, and bare soil. After classification, the vegetation, water, and bare soil classes were merged into a non-urban class. A majority filter with a $3 \times 3$ window was applied to the classification results to reduce isolated pixels. Finally, we up-scaled the $28.5 \mathrm{~m}$ pixel classification results to $1 \mathrm{~km}$ pixels. First, we determined the percentage of $28.5 \mathrm{~m}$ urban pixels inside each $1 \mathrm{~km}$ pixel. If it was larger than $50 \%$, the pixel was considered urban, otherwise the pixel was non-urban. The urban classification results of the $1 \mathrm{~km}$ pixels were generated for each city under consideration.

Since the locally optimized threshold method performs well using DMSP/OLS NTL data [29,35,38], we applied it to the DMSP/OLS NTL image to extract the urban extent for comparison. In this study, we determined an optimal local DN threshold for each city by matching the urban area derived from DMSP/OLS NTL data to the urban area from the Landsat TM/ETM+ classification, as closely as possible.

We used the confusion matrix [76] as a thematic accuracy assessment to quantify the performance of the different methods. For each urban extent mapping result, the accuracy assessment includes the producer's accuracy, user's accuracy, overall accuracy, and kappa coefficient. Although the kappa coefficient has known problems when used to assess accuracies in remote sensing applications [77,78], it can evaluate the classifications more thoroughly than the overall accuracy. Considering the kappa coefficient was widely used in accuracy assessment for regional urban extent mapping using DMSP/OLS NTL and related data [35-37], the kappa coefficient is also used in this study. Additionally, to evaluate the performance in terms of the geometric properties, we used a discrepancy measure called the average differences of shape index [79] to compare the proposed methods with the locally optimized method. Discrepancy measures of the geometric properties were originally used to evaluate image segmentation and classification results for very high resolution images [79]. The average difference of shape index quantitatively compares the shape indexes of urban objects produced by the proposed method with the reference data.

Urban built-up areas derived from existing urban statistical data [69] were also used to assess the performance of the proposed methods. We computed and analyzed the correlation coefficients comparing the urban built-up areas from the statistical data with those extracted from the proposed method.

\section{Results}

Figure 4 shows training samples selected with and without the MODIS LST data. The training samples for the urban class selected using the DMSP/OLS NLT and MODIS NDVI data include water pixels, which will affect the classification results. These water pixels were not present when using the combination of the DMSP/OLS NLT, MODIS NDVI, and LST data. Thus, the combination of three datasets provides more reliable training samples than only using the DMSP/OLS NTL and MODIS NDVI data. 


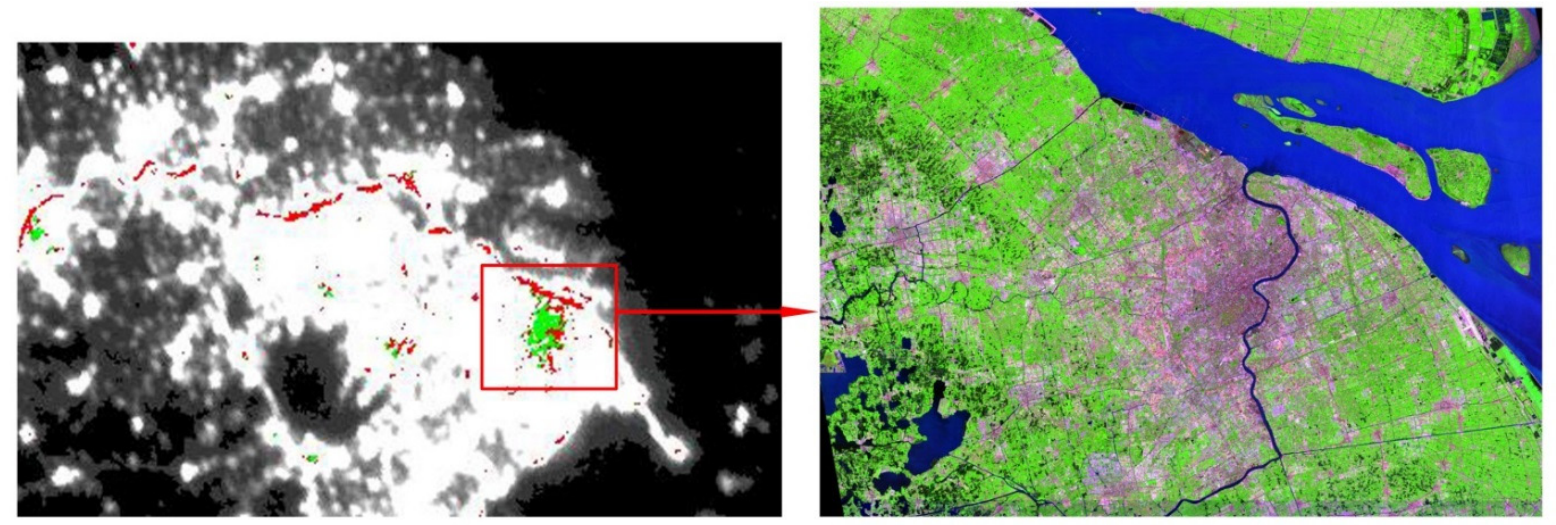

(a)

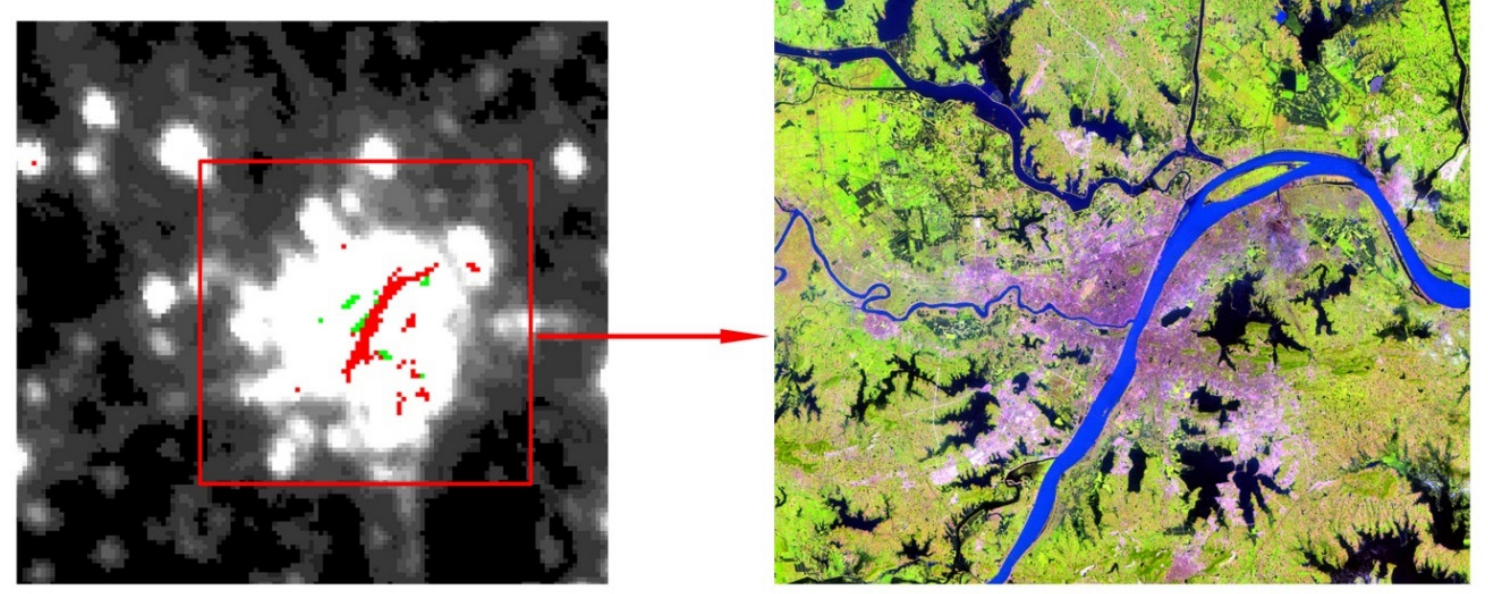

(b)

Figure 4. Training samples selected using different data combinations for Shanghai (a) and Wuhan (b). The left images are NTL data. The right images are Landsat TM images (band 7, 4, 3 as R, G, B). The green points represent the training samples selected from three datasets (also from the NTL data and NDVI data), and the red ones represent the samples selected only from the NTL data and NDVI data. The red points include the pixels of river.

The classification results from Landsat TM/ETM+ data that are used as a reference map were validated using test samples of typical urban areas, and were over $80 \%$ of overall accuracy.

Figure 5 shows the DMSP/OLS NTL images, reference urban area results from Landsat TM/ETM+ images $(1 \mathrm{~km})$, and the urban extent mapping results extracted using different methods, for six cities of different population sizes. The reference data contain many details, including many isolated pixels (Figure 5b). However, the urban areas generated by all the methods using coarse multi-sensor data are relatively continuous, with only a few isolated urban pixels (Figure $5 \mathrm{c}-\mathrm{g}$ ). The local-optimized threshold method (Figure 5c) accurately extracted the main urban areas, but omitted some pixels from small urban areas around the main urban areas. However, the OCSVM classification results using the combination of DMSP/OLS NTL and MODIS NDVI data, and the combination of all three datasets were very similar. The main and small urban areas were accurately extracted (Figure 5d,f). In particular, the classification results based on the combination of all three datasets performed best for the small cities, i.e., Xuchang and Hengshui (Figure 5f). However, the classification results from the combination of DMSP/OLS NTL 
and MODIS LST data were homogeneous, but significantly overestimated the urban extent compared with the reference data (Figure 5e). Table 1 shows the average differences in the shape indexes between the extracted results and reference data. The proposed methods using different data combinations had lower discrepancy values for the average shape index differences than the locally optimized method, which indicates that the proposed methods outperformed the locally optimized method in terms of the shape. This also agrees with the visual comparison in Figure 5.

The classification results using different data combinations and from the locally optimized threshold method are summarized in Table 2. The locally optimized threshold method performed best for 11 out of 55 cities, including six big cities, four medium cities, and one small city. For the big cities, the locally optimized threshold method generally achieved higher kappa values than other methods. However, for small cities (especially in middle of China), the locally optimized threshold methods produced lower kappa values than the other methods. The locally optimized threshold method omitted a large number of urban pixels for these small cities.

(a)

(b)

(c)

(d)

(e)

(f)
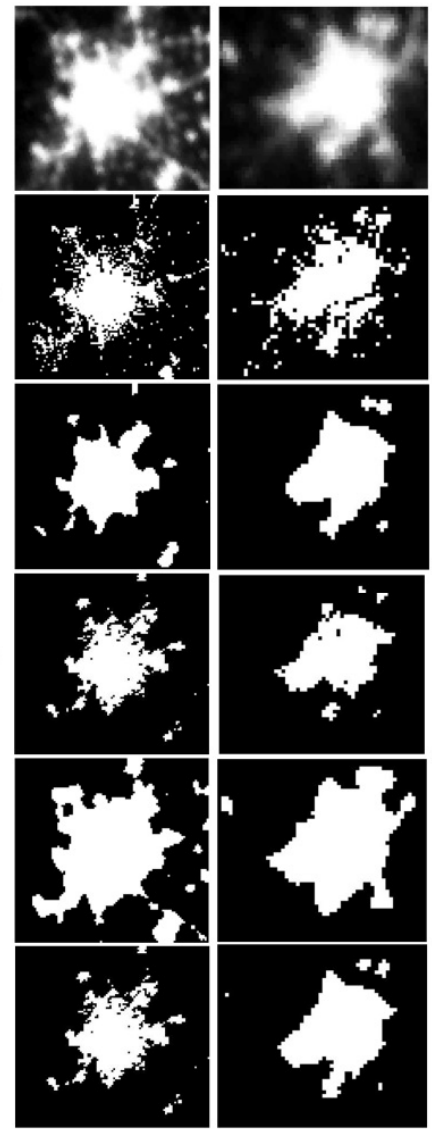

Beijing
Shenyang

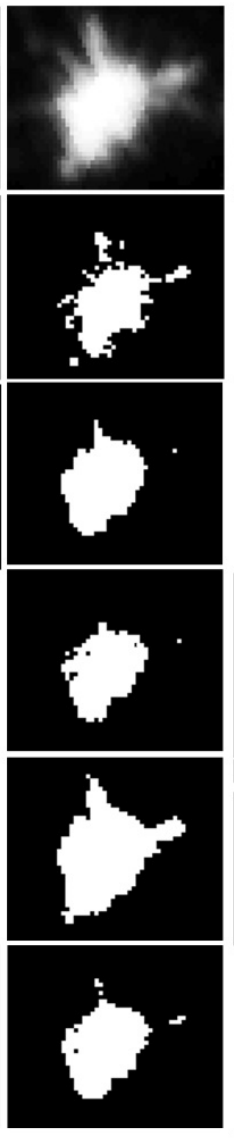

Hefei

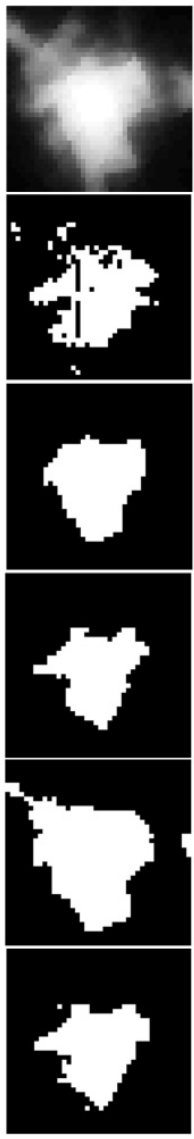

Changsha
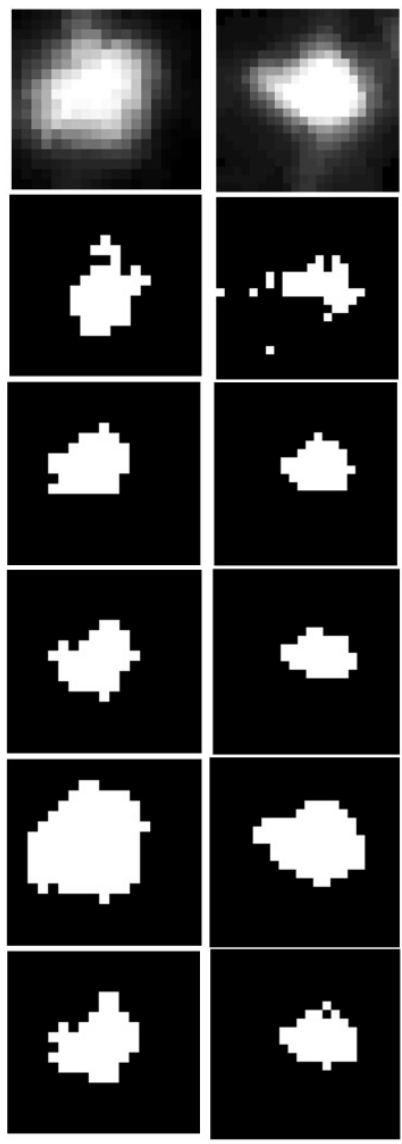

Xuchang Hengshui

Figure 5. Urban area extraction results from different methods. (a) DMSP/OLS NTL images of selected cities; (b) The reference data from Landsat ETM + classification; (c) Local-optimized threshold method; (d) OCSVM result with combination of DMSP/OLS NTL data and MODIS NDVI data; (e) OCSVM result with the combination of DMSP/OLS NTL data and MODIS LST data; (f) OCSVM result with combination of DMSP/OLS NTL data, MODIS NDVI and LST data. 
Table 1. Average difference in shape index between the extracted results from different methods and the reference data.

\begin{tabular}{ccccc}
\hline & $\begin{array}{c}\text { Local-Optimized } \\
\text { Threshold Method }\end{array}$ & $\begin{array}{c}\text { OCSVM Classification } \\
\text { with NTL + NDVI }\end{array}$ & $\begin{array}{c}\text { OCSVM Classification } \\
\text { with NTL + LST }\end{array}$ & $\begin{array}{c}\text { OCSVM Classification } \\
\text { with NTL + NDVI + LST }\end{array}$ \\
\hline Beijing & 0.1642 & 0.1312 & 0.1460 & 0.1183 \\
Shenyang & 0.2458 & 0.2190 & 0.1734 & 0.1972 \\
Hefei & 0.1910 & 0.1169 & 0.0820 & 0.1712 \\
Changsha & 0.3620 & 0.2023 & 0.2611 & 0.2193 \\
Xuchang & 0.1846 & 0.0815 & 0.1963 & 0.0785 \\
Hengshui & 0.1725 & 0.2196 & 0.2087 & 0.1196 \\
\hline
\end{tabular}

The OCSVM classification with DMSP/OLS NTL and MODIS NDVI data performed best for 16 cities, including five big cities, seven medium cities, and four small cities (Table 2). The OCSVM classification based on this data combination produced higher kappa values than the locally optimized threshold method for 37 out of 55 cities. Additionally, for big cities, the OCSVM classification based on this data combination performed as well as the locally optimized threshold method. For medium and small cities, the OCSVM classification based on NTL and NDVI data outperformed the locally optimized threshold method. Specifically, the OCSVM classification based on this data combination achieved higher kappa values for 13 out of 18 medium cities and 14 out of 18 small cities, when compared with the locally optimized threshold method. Thus, the MODIS NDVI data provided additional discriminative information for extracting the urban extent.

The OCSVM classification based on the combination of DMSP/OLS NTL and LST data obtained a relatively low accuracies for big and medium cities, because it overestimated urban areas, when compared with the other classification results (Table 2). However, the combination of NTL and LST data outperformed the other data combinations and the locally optimized threshold method for small urban areas (e.g., Macau). This may be because the temperature gradient between urban and rural areas is not as obvious as the difference in vegetation coverage over urban and rural areas. Additionally, LST and NTL data have similar fluctuations, whereas NTL and NDVI data had opposite fluctuations (Figure 3).

The OCSVM classification based on the combination of DMSP/OLS NTL, MODIS NDVI, and LST data achieved the highest kappa values for 21 out of 55 cities, including five big cities, five medium cities, and 11 small cities (Table 2). The OCSVM classification based on this combination produced higher kappa values than the locally optimized threshold method for 38 out of 55 cities (Table 2). In particular, for small cities, the classification accuracies (in terms of kappa) were significantly improved using this combination. For example, the kappa value of the combination of three datasets for Xuchang (a small city) is 0.72 , whereas the kappa value produced using the local-optimized threshold method is only 0.49 . 
Table 2. Accuracy assessment of different methods of extracting urban areas for 55 cities.

\begin{tabular}{|c|c|c|c|c|c|c|c|c|c|c|}
\hline & \multirow{2}{*}{ City } & \multirow{2}{*}{ Population (Thousand) } & \multicolumn{2}{|c|}{ Local-Optimized Threshold } & \multicolumn{2}{|c|}{ OCSVM NTL + NDVI } & \multicolumn{2}{|c|}{ OCSVM NTL + LST } & \multicolumn{2}{|c|}{ OCSVM NTL + NDVI + LST } \\
\hline & & & OA (\%) & Kappa & OA (\%) & Kappa & OA (\%) & Kappa & OA (\%) & Kappa \\
\hline \multirow{19}{*}{$\begin{array}{c}\text { The first group: } \\
\text { Big cities }\end{array}$} & Shanghai & $11,511.9$ & 89.01 & 0.6222 & 88.16 & 0.6357 & 77.22 & 0.4623 & 87.31 & 0.6286 \\
\hline & Beijing & 8792.8 & 94.89 & 0.6521 & 94.48 & 0.6665 & 88.58 & 0.5062 & 93.94 & 0.6531 \\
\hline & Hong Kong & 6857.1 & 79.95 & 0.5119 & 82.22 & 0.5842 & 71.31 & 0.4058 & 84.1 & 0.6106 \\
\hline & Chongqing & 5966.9 & 87.08 & 0.6018 & 90.22 & 0.6529 & 80.59 & 0.5239 & 89.46 & 0.6469 \\
\hline & Tianjin & 5400.2 & 90.68 & 0.5548 & 91.74 & 0.6002 & 86.69 & 0.5174 & 91.87 & 0.6176 \\
\hline & Guangzhou & 4909.5 & 78.54 & 0.5155 & 68.94 & 0.4188 & 54.06 & 0.2345 & 65.95 & 0.3812 \\
\hline & Nanjing & 4470.4 & 87.73 & 0.6466 & 89.17 & 0.7011 & 76.48 & 0.4891 & 88.56 & 0.6969 \\
\hline & Wuhan & 4446.4 & 91.85 & 0.5495 & 92.15 & 0.5668 & 86.79 & 0.4642 & 91.55 & 0.5646 \\
\hline & Shenyang & 4441.8 & 90.31 & 0.7006 & 90.56 & 0.6908 & 85.71 & 0.6314 & 90.96 & 0.7237 \\
\hline & Chengdu & 3802.8 & 91.99 & 0.7787 & 90.63 & 0.6748 & 85.76 & 0.658 & 92.91 & 0.7682 \\
\hline & Harbin & 3413 & 94.86 & 0.6095 & 92.52 & 0.5925 & 84.72 & 0.4095 & 91.82 & 0.5697 \\
\hline & Xi'an & 3182 & 85.52 & 0.619 & 75.67 & 0.4947 & 55.05 & 0.2445 & 73.4 & 0.4615 \\
\hline & Jinan & 2770.2 & 95.23 & 0.7222 & 90.74 & 0.599 & 78.92 & 0.3701 & 89.36 & 0.5619 \\
\hline & Taipei & 2632.242 & 90.85 & 0.5528 & 90.51 & 0.6239 & 78.23 & 0.4038 & 89.16 & 0.5982 \\
\hline & Hangzhou & 2564.2 & 86.61 & 0.5726 & 84.68 & 0.3835 & 84.32 & 0.5739 & 85.71 & 0.4558 \\
\hline & Changchun & 2508.5 & 93.96 & 0.783 & 94.68 & 0.8154 & 90.69 & 0.7164 & 94.18 & 0.8048 \\
\hline & Shijiazhuang & 2313.5 & 94.88 & 0.6859 & 95.12 & 0.713 & 86.87 & 0.5031 & 94.92 & 0.72 \\
\hline & Taiyuan & 2276.9 & 89.04 & 0.564 & 89.44 & 0.5606 & 88.19 & 0.5883 & 89.44 & 0.5797 \\
\hline & Wuxi & 2186.3 & 88.11 & 0.5963 & 86.21 & 0.5946 & 61.31 & 0.2774 & 83.4 & 0.5544 \\
\hline \multirow{8}{*}{$\begin{array}{c}\text { The second } \\
\text { group:Medium } \\
\text { cities }\end{array}$} & Shenzhen & 1968.3 & 79.04 & 0.4444 & 81.23 & 0.5768 & 69.73 & 0.3966 & 79.92 & 0.5523 \\
\hline & Zhengzhou & 1932.6 & 92.43 & 0.5903 & 92.78 & 0.6312 & 85.38 & 0.472 & 92.7 & 0.6426 \\
\hline & Fuzhou & 1817.2 & 91.07 & 0.5376 & 90.57 & 0.457 & 91.45 & 0.584 & 91.23 & 0.5151 \\
\hline & Changsha & 1798.9 & 92.72 & 0.7256 & 93.65 & 0.7475 & 88.68 & 0.6533 & 94.16 & 0.7758 \\
\hline & Nanchang & 1737.5 & 87.59 & 0.6183 & 88.11 & 0.6532 & 80.73 & 0.5221 & 85.59 & 0.6035 \\
\hline & Lanzhou & 1723.1 & 94.88 & 0.5889 & 85.93 & 0.4071 & 85.12 & 0.3912 & 85.32 & 0.3951 \\
\hline & Kunming & 1720.7 & 93.7 & 0.6385 & 94.64 & 0.6804 & 91.25 & 0.572 & 94.35 & 0.673 \\
\hline & Hefei & 1605.4 & 94.2 & 0.7135 & 94.97 & 0.7358 & 91.15 & 0.6403 & 94.9 & 0.7466 \\
\hline
\end{tabular}


Table 2. Cont

\begin{tabular}{|c|c|c|c|c|c|c|c|c|c|c|}
\hline & \multirow{2}{*}{ City } & \multirow{2}{*}{ Population (Thousand) } & \multicolumn{2}{|c|}{ Local-Optimized Threshold } & \multicolumn{2}{|c|}{ OCSVM NTL + NDVI } & \multicolumn{2}{|c|}{ OCSVM NTL + LST } & \multicolumn{2}{|c|}{ OCSVM NTL + NDVI + LST } \\
\hline & & & OA (\%) & Kappa & OA (\%) & Kappa & OA (\%) & Kappa & OA (\%) & Kappa \\
\hline \multirow{10}{*}{$\begin{array}{c}\text { The second } \\
\text { group:Medium } \\
\text { cities }\end{array}$} & Urumchi & 1576.6 & 97.5 & 0.6572 & 94.65 & 0.5205 & 93.58 & 0.4735 & 94.36 & 0.5084 \\
\hline & Zibo & 1557.4 & 91.19 & 0.3173 & 90.6 & 0.4199 & 79.94 & 0.28 & 89.48 & 0.4092 \\
\hline & Guiyang & 1513 & 93.02 & 0.6505 & 93.75 & 0.535 & 92.69 & 0.6752 & 93.94 & 0.5994 \\
\hline & Suzhou & 1501.4 & 88.02 & 0.5665 & 83.95 & 0.5585 & 64.15 & 0.3053 & 81.2 & 0.5207 \\
\hline & Nanning & 1308.1 & 97.5 & 0.7443 & 98.37 & 0.8138 & 96.14 & 0.6618 & 98.18 & 0.8044 \\
\hline & Fushun & 1260.2 & 89.15 & 0.4947 & 90.93 & 0.5456 & 84.42 & 0.4758 & 90.73 & 0.5856 \\
\hline & Ningbo & 1257.6 & 89.76 & 0.5356 & 88.54 & 0.583 & 66.46 & 0.2804 & 85.67 & 0.5402 \\
\hline & Handan & 1245.8 & 89.23 & 0.5867 & 90.46 & 0.6425 & 84.15 & 0.5556 & 91.69 & 0.6979 \\
\hline & Changzhou & 1115.9 & 89.71 & 0.5398 & 88.24 & 0.5656 & 65.02 & 0.2553 & 85.35 & 0.5208 \\
\hline & Xiamen & 1092.4 & 79.6 & 0.3562 & 81.44 & 0.4724 & 72.24 & 0.3222 & 81.17 & 0.4622 \\
\hline \multirow{18}{*}{$\begin{array}{c}\text { The third group: } \\
\text { Small cities }\end{array}$} & Hengyang & 966 & 91.15 & 0.5698 & 92.8 & 0.573 & 90.74 & 0.5691 & 93.42 & 0.6331 \\
\hline & Xiangfan & 953.4 & 94.14 & 0.6961 & 94.14 & 0.6642 & 92.03 & 0.6474 & 94.41 & 0.7005 \\
\hline & Baoding & 927.5 & 93.24 & 0.4964 & 93.38 & 0.5385 & 89.32 & 0.4671 & 93.48 & 0.5598 \\
\hline & Xining & 909.8 & 98.99 & 0.2606 & 98.53 & 0.3442 & 98.14 & 0.3033 & 98.38 & 0.3338 \\
\hline & Haikou & 897.7 & 84.88 & 0.4432 & 88.37 & 0.5052 & 86.21 & 0.5187 & 88.27 & 0.5168 \\
\hline & Hohhot & 842.3 & 94.64 & 0.7457 & 77.74 & 0.4046 & 68.45 & 0.2963 & 74.64 & 0.3679 \\
\hline & Yinchuan & 711.9 & 95.39 & 0.5424 & 94.99 & 0.5603 & 92.01 & 0.4401 & 94.3 & 0.5324 \\
\hline & Xinxiang & 711.9 & 90.48 & 0.649 & 91.1 & 0.6999 & 69.77 & 0.3438 & 89.65 & 0.6675 \\
\hline & Yichang & 702.2 & 96.8 & 0.5568 & 97.22 & 0.5484 & 95.75 & 0.5455 & 97.22 & 0.5813 \\
\hline & Anyang & 688 & 87.93 & 0.6567 & 91.33 & 0.7547 & 80.5 & 0.5586 & 91.33 & 0.7594 \\
\hline & Guilin & 629.1 & 98.47 & 0.5378 & 98.27 & 0.2551 & 98.49 & 0.5896 & 98.3 & 0.2897 \\
\hline & Kaifeng & 592.4 & 87.2 & 0.3727 & 90.13 & 0.4768 & 85.6 & 0.4206 & 89.73 & 0.4896 \\
\hline & Xianyang & 545.3 & 75 & 0.3226 & 78.1 & 0.4604 & 55.48 & 0.2367 & 79.52 & 0.5127 \\
\hline & Macau & 513.4 & 59.62 & 0.1817 & 66.35 & 0.3142 & 75.96 & 0.5138 & 74.04 & 0.467 \\
\hline & Cangzhou & 492.6 & 89.38 & 0.3986 & 91.72 & 0.5396 & 85.52 & 0.4302 & 91.59 & 0.5649 \\
\hline & Xuchang & 401.5 & 89.18 & 0.4927 & 92.69 & 0.6573 & 81.58 & 0.4654 & 93.57 & 0.7239 \\
\hline & Hengshui & 298.1 & 97.28 & 0.5485 & 97.5 & 0.5776 & 96.03 & 0.5578 & 97.61 & 0.6275 \\
\hline & Lhasa & 211.4 & 96.74 & 0.6312 & 96.78 & 0.6617 & 96.34 & 0.6306 & 96.6 & 0.6489 \\
\hline
\end{tabular}

Note: The bold text in the table represents the maximum value of each row. 
Table 3 shows the correlation coefficients between urban areas extracted using different methods and those from the statistical data for cities of different sizes. For big cities, the urban areas derived from the proposed methods using different data combinations were highly correlated with urban built-up areas determined using the statistical data [69], with correlation coefficients greater than 0.8. In particular, the urban areas extracted using the combination of all three datasets were most correlated with urban built-up areas derived from the statistical data [69]. For medium cities, the correlation coefficients comparing the urban areas extracted using all the methods with the urban areas derived from the statistical data were greater than 0.92 , with the exception of the NTL and LST combination (which had a correlation coefficient lower than 0.9). For small cities, the correlation coefficients of the proposed method $(>0.7)$ were much larger than those of the locally optimized threshold method $(<0.55)$. We found that the urban areas derived from the combination of three datasets were highly correlated with urban built-up areas derived from the statistical data. The highest correlation was between the urban areas that were based on the combination of the three datasets and the statistically derived built-up areas, in two cases (i.e., big cities and small cities). In general, the proposed methods using different data combinations performed better than (or were at least comparable to) the locally optimized threshold method in terms of the correlation with urban areas derived from statistical data.

Table 3. Correlation coefficients between the results from different methods and statistical built-up areas.

\begin{tabular}{ccccc}
\hline & Local-Optimized Threshold & $\begin{array}{c}\text { OCSVM NTL + NDVI + } \\
\text { LST }\end{array}$ & $\begin{array}{c}\text { OCSVM NTL + } \\
\text { NDVI }\end{array}$ & $\begin{array}{c}\text { OCSVM NTL } \\
+ \text { LST }\end{array}$ \\
\hline Big cities & 0.7700 & 0.8190 & 0.8127 & 0.8128 \\
Medium cities & 0.9321 & 0.9175 & 0.9269 & 0.8763 \\
Small cities & 0.5439 & 0.7267 & 0.7139 & 0.6914 \\
\hline
\end{tabular}

\section{General Discussion}

The results presented above demonstrated that DMSP/OLS NTL data, MODIS NDVI and LST data provide different but complementary information sources to quantify properties of urban areas, as shown in Figure 3. The urban extent mapping results showed that the OCSVM classification with combination of DMSP/OLS NTL, MODIS NDVI and LST data outperformed the locally optimized threshold method, a widely used urban extent mapping method.

Although the NDVI data are a valuable information source for improving the urban extent mapping using DMSP/OLS NTL data alone (e.g., [16,35]), it is found from this study that MODIS LST data also provided additional discriminative information. For example, the inclusion of LST and NDVI data achieved the best accuracy in the small cities. The LST data strengthened the differences between urban and non-urban areas, because of the temperature difference between urban and rural areas. It should be mentioned that the MODIS LST data were not only used in the classification, but were also used to select training samples for classifications of all data combinations. The combination of three datasets provides more reliable training samples than the use of the DMSP/OLS NTL and MODIS NDVI data only, as shown in Figure 4. Thus, it is understandable that the classification using DMSP/OLS NTL and MODIS NDVI data produced similar results to the classification using all three datasets for medium-size 
cities and large cities, since MODIS LST data were also used to select the training samples in classification using DMSP/OLS NTL and MODIS NDVI data.

The experimental results also show that the OCSVM classification provided an effective and efficient method of classifying multi-sensor data combinations for extracting urban areas at regional scale. Compared with traditional two-class SVM classification methods which require training samples from both classes [35,37], the OCSVM does not require samples from complex backgrounds with significant variability (non-urban areas). Moreover, the OCSVM can focus on the urban areas, the only class of interest. In terms of mapping accuracy, the proposed OCSVM based method achieved generally better results than the locally optimized threshold method, whereas the existing two-class SVM classification-based method [35] produced extraction accuracies comparable to the locally optimized threshold method. Thus, from these observations, the proposed OCSVM based method is more effective than the two-class SVM-based method (i.e., [35]) in urban extent mapping at regional scale.

It is also worth noting that the locally optimized threshold was generated by comparing reference data for each city, the method heavily relies on the availability of high-resolution data of the whole study area and requires extensive and tedious work. In contrast, the proposed OCSVM-based method is not very dependent on the high resolution images and is more efficient in extracting the urban extent.

There are still some limitations with the proposed method. The inclusion of LST and NDVI data in urban extent extraction may fail in some places. Since our proposed method is based on the assumption that there are significant differences in vegetation and temperature features between urban and non-urban areas, the assumption will be invalid for the regions where there are no vegetation or little vegetation surrounding urban areas and there is no significant difference between urban and non-urban areas. Thus, the inclusion of LST and NDVI data in urban extent extraction will fail in these regions. For our study area, we found that the proposed method failed only in few cities. For example, for cities in arid or semi-arid regions (e.g., Lanzhou), there is more vegetation in urban areas (since vegetation is mainly planted on artificial irrigation systems) and the temperatures of urban areas are even lower than those of non-urban areas. However, the inclusion of LST and NDVI data is generally effective for regions with rapid urbanization, where the 'Urban Heat Island' effect is obvious and the fractional impervious surface area and the fractional vegetation cover vary inversely in urban areas [41]. Considering the above discussions, further investigation is required to explore new and more sophisticated methods to solve these problems.

\section{Conclusions}

In this study, MODIS LST data were combined with DMSP/OLS NTL and MODIS NDVI data to quantify and extract urban areas at regional scale. The OCSVM, a one-class classifier, were adopted to classify different data combinations of three datasets. The proposed method was compared with the locally optimized threshold method, a widely used method. The experimental results demonstrate that DMSP/OLS NTL, MODIS NDVI, and LST data provide different but complementary information sources to quantify the urban extent at regional scale. The combination of DMSP/OLS NTL, MODIS NDVI, and LST data was the best data combination for OCSVM classification, and outperformed the locally-optimized threshold method. Therefore, the OCSVM classification of combination of 
DMSP/OLS NTL, MODIS LST, and NDVI data is an effective and efficient method for extracting the urban extent at regional scale, and can be applied to other study areas.

\section{Acknowledgments}

This work was funded by National Science Foundation of China (Grant Number 41371329). We would like to thank anonymous reviewers for their constructive comments, which greatly improved the quality of our manuscript.

\section{Author Contributions}

Xiya Zhang and Peijun Li conceived and designed the paper. Xiya Zhang mainly analyzed the data and wrote the paper. Peijun Li designed and revised the paper. Cai Cai helped produce partial reference data using Landsat TM/ETM+ images.

\section{Conflicts of Interest}

The authors declare no conflict of interest.

\section{References}

1. Alberti, M. The effects of urban patterns on ecosystem function. Int. Reg. Sci. Rev. 2005, 28, 168-192.

2. Grimmond, S.U.E. Urbanization and global environmental change: Local effects of urban warming. Geogr. J. 2007, 173, 83-88.

3. Johnson, M.P. Environmental impacts of urban sprawl: A survey of the literature and proposed research agenda. Environ. Plan. A 2001, 33, 717-735.

4. Shukla, V.; Parikh, K. The environmental consequences of urban growth: Cross national perspectives on economic development, air pollution, and city size. Urban Geogr. 1992, 13, 422-449.

5. Foley, J.A.; DeFries, R.; Asner, G.P.; Barford, C.; Bonan, G.; Carpenter, S.R.; Chapin, F.S.; Coe, M.T.; Daily, G.C.; Gibbs, H.K.; et al. Global consequences of land use. Science 2005, 309, 570-574.

6. Kasimu, A.; Tateishi, R.; Hoan, N.T. Global urban characterization using population density, DMSP and MODIS data. In Proceedings of the Urban Remote Sensing Event, 2009 Joint, Shanghai, China, 20-22 May 2009; pp. 1-7.

7. McKinney, M. Effects of urbanization on species richness: A review of plants and animals. Urban Ecosyst. 2008, 11, 161-176.

8. Small, C.; Pozzi, F.; Elvidge, C.D. Spatial analysis of global urban extent from DMSP-OLS night lights. Remote Sens. Environ. 2005, 96, 277-291.

9. Meyer, W.B.; Turner, B.L. Human population growth and global land-use/cover change. Annu. Rev. Ecol. Syst. 1992, 23, 39-61.

10. Ewing, R.; Rong, F. The impact of urban form on U.S. residential energy use. Hous. Policy Debate 2008, 19, 1-30.

11. Jensen, J.R.; Cowen, D.C. Remote sensing of urban/suburban infrastructure and socio-economic attributes. Photogram. Eng. Remote Sens. 1999, 65, 611-622. 
12. Tian, G.; Liu, J.; Xie, Y.; Yang, Z.; Zhuang, D.; Niu, Z. Analysis of spatio-temporal dynamic pattern and driving forces of urban land in China in 1990s using TM images and GIS. Cities 2005, 22, $400-410$.

13. Liu, J.; Liu, M.; Zhuang, D.; Zhang, Z.; Deng, X. Study on spatial pattern of land-use change in China during 1995-2000. Sci. China Ser. D Earth Sci. 2003, 46, 373-384.

14. Liu, J.; Liu, M.; Tian, H.; Zhuang, D.; Zhang, Z.; Zhang, W.; Tang, X.; Deng, X. Spatial and temporal patterns of China's cropland during 1990-2000: An analysis based on landsat TM data. Remote Sens. Environ. 2005, 98, 442-456.

15. Liu, J.; Zhang, Z.; Xu, X.; Kuang, W.; Zhou, W.; Zhang, S.; Li, R.; Yan, C.; Yu, D.; Wu, S. Spatial patterns and driving forces of land use change in China during the early 21 st century. J. Geogr. Sci. 2010, 20, 483-494.

16. Lu, D.; Tian, H.; Zhou, G.; Ge, H. Regional mapping of human settlements in southeastern China with multisensor remotely sensed data. Remote Sens. Environ. 2008, 112, 3668-3679.

17. Elvidge, C.; Baugh, K.; Hobson, V.; Kihn, E.; Kroehl, H.; Davis, E.; Cocero, D. Satellite inventory of human settlements using nocturnal radiation emissions: A contribution for the global toolchest. Glob. Chang. Biol. 1997, 3, 387-395.

18. Sudhira, H.S.; Ramachandra, T.V.; Jagadish, K.S. Urban sprawl: Metrics, dynamics and modelling using GIS. Int. J. Appl. Earth Obs. Geoinf. 2004, 5, 29-39.

19. Bartholomé, E.; Belward, A.S. GLC2000: A new approach to global land cover mapping from earth observation data. Int. J. Remote Sens. 2005, 26, 1959-1977.

20. Gamba, P.; Herold, M. Global Mapping of Human Settlement: Experiences, Datasets, and Prospects; CRC Press: Boca Raton, FL, USA, 2010.

21. Schneider, A.; Friedl, M.A.; Potere, D. Mapping global urban areas using MODIS $500 \mathrm{~m}$ data: New methods and datasets based on "urban ecoregions". Remote Sens. Environ. 2010, 114, 1733-1746.

22. Zhang, Q.; Seto, K.C. Mapping urbanization dynamics at regional and global scales using multi-temporal DMSP/OLS nighttime light data. Remote Sens. Environ. 2011, 115, 2320-2329.

23. Arino, O.; Gross, D.; Ranera, F.; Bourg, L.; Bicheron, P.; Latham, J.; di Gregorio, A.; Brockman, C.; Leroy, M.; Witt, R.; et al. Globcover: ESA Service for global land cover from MERIS. In Proceedings of the IEEE International Geoscience and Remote Sensing Symposium, Barcelona, Spain, 23-28 July 2007; pp. 2412-2415.

24. Imhoff, M.L.; Lawrence, W.T.; Stutzer, D.C.; Elvidge, C.D. A technique for using composite DMSP/OLS "city lights" satellite data to map urban area. Remote Sens. Environ. 1997, 61, 361-370.

25. Elvidge, C.D.; Sutton, P.C.; Tuttle, B.T.; Ghosh, T.; Baugh, K.E. Global urban mapping based on nighttime lights. In Global Mapping of Human Settlement; CRC Press: Boca Raton, FL, USA, 2009.

26. Milesi, C.; Elvidge, C.D.; Nemani, R.R.; Running, S.W. Assessing the environmental impacts of human settlements using satellite data. Manag. Environ. Qual. 2003, 14, 99-107.

27. Elvidge, C.D.; Baugh, K.E.; Dietz, J.B.; Bland, T.; Sutton, P.C.; Kroehl, H.W. Radiance calibration of DMSP-OLS low-light imaging data of human settlements. Remote Sens. Environ. 1999, 68, 77-88.

28. Elvidge, C.D.; Imhoff, M.L.; Baugh, K.E.; Hobson, V.R.; Nelson, I.; Safran, J.; Dietz, J.B.; Tuttle, B.T. Night-time lights of the world: 1994-1995. ISPRS J. Photogramm. Remote Sens. 2001, 56, 81-99.

29. Henderson, M.; Yeh, E.T.; Gong, P.; Elvidge, C.; Baugh, K. Validation of urban boundaries derived from global night-time satellite imagery. Int. J. Remote Sens. 2003, 24, 595-609. 
30. Sutton, P.C. A scale-adjusted measure of "urban sprawl" using nighttime satellite imagery. Remote Sens. Environ. 2003, 86, 353-369.

31. Amaral, S.; Câmara, G.; Monteiro, A.M.V.; Quintanilha, J.A.; Elvidge, C.D. Estimating population and energy consumption in Brazilian Amazonia using DMSP night-time satellite data. Comput. Environ. Urban Syst. 2005, 29, 179-195.

32. Elvidge, C.D.; Cinzano, P.; Pettit, D.R.; Arvesen, J.; Sutton, P.; Small, C.; Nemani, R.; Longcore, T.; Rich, C.; Safran, J.; et al. The nightsat mission concept. Int. J. Remote Sens. 2007, 28, 2645-2670.

33. Owen, T.W. Using DMSP-OLS light frequency data to categorize urban environments associated with US climate observing stations. Int. J. Remote Sens. 1998, 19, 3451-3456.

34. Sutton, P.; Roberts, D.; Elvidge, C.; Baugh, K. Census from heaven: An estimate of the global human population using night-time satellite imagery. Int. J. Remote Sens. 2001, 22, 3061-3076.

35. Cao, X.; Chen, J.; Imura, H.; Higashi, O. A SVM-based method to extract urban areas from DMSP-OLS and SPOT VGT data. Remote Sens. Environ. 2009, 113, 2205-2209.

36. Pandey, B.; Joshi, P.K.; Seto, K.C. Monitoring urbanization dynamics in India using DMSP/OLS night time lights and SPOT-VGT data. Int. J. Appl. Earth Obs. Geoinf. 2013, 23, 49-61.

37. Yang, Y.; He, C.; Zhang, Q.; Han, L.; Du, S. Timely and accurate national-scale mapping of urban land in China using defense meteorological satellite program's operational linescan system nighttime stable light data. J. Appl. Remote Sens. 2013, 7, 073535.

38. Zhou, Y.; Smith, S.J.; Elvidge, C.D.; Zhao, K.; Thomson, A.; Imhoff, M. A cluster-based method to map urban area from DMSP/OLS nightlights. Remote Sens. Environ. 2014, 147, 173-185.

39. Gallo, K.P.; Tarpley, J.D.; McNab, A.L.; Karl, T.R. Assessment of urban heat islands: A satellite perspective. Atmos. Res. 1995, 37, 37-43.

40. Rotem-Mindali, O.; Michael, Y.; Helman, D.; Lensky, I.M. The role of local land-use on the urban heat island effect of Tel AVIV as assessed from satellite remote sensing. Appl. Geogr. 2015, 56, 145-153.

41. Dewan, A.M.; Corner, R.J. Impact of land use and land cover changes on urban land surface temperature. In Dhaka Megacity; Springer: Berlin, Germany, 2014; pp. 219-238.

42. Gallo, K.P.; McNab, A.L.; Karl, T.R.; Brown, J.F.; Hood, J.J.; Tarpley, J.D. The use of NOAA AVHRR data for assessment of the urban heat island effect. J. Appl. Meteorol. 1993, 32, 899-908.

43. Lu, D.; Weng, Q. Use of impervious surface in urban land-use classification. Remote Sens. Environ. 2006, 102, 146-160.

44. Yang, F.; Matsushita, B.; Yang, W.; Fukushima, T. Mapping the human footprint from satellite measurements in Japan. ISPRS J. Photogramm. Remote Sens. 2014, 88, 80-90.

45. Melesse, A.M.; Graham, W.D.; Jordan, J.D. Spatially distributed watershed mapping and modeling: Thermal maps and vegetation indices to enhance land cover and surface microclimate mapping (part 1). J. Spat. Hydrol. 2003, 3, 1-29.

46. Julien, Y.; Sobrino, J.A.; Jiménez-Muñoz, J.C. Land use classification from multitemporal landsat imagery using the yearly land cover dynamics (YLCD) method. Int. J. Appl. Earth Obs. Geoinf. 2011, 13, 711-720.

47. Sobrino, J.; Raissouni, N.; Lobo, A. Monitoring the Iberian peninsula land cover using NOAA-AVHRR data. Phys. Meas. Signat. Remote Sens. 1997, 787-794.

48. Lambin, E.; Ehrlich, D. Combining vegetation indices and surface temperature for land-cover mapping at broad spatial scales. Remote Sens. 1995, 16, 573-579. 
49. Lambin, E.; Ehrlich, D. The surface temperature-vegetation index space for land cover and land-cover change analysis. Int. J. Remote Sens. 1996, 17, 463-487.

50. Hao, R.; Yu, D.; Sun, Y.; Cao, Q.; Liu, Y.; Liu, Y. Integrating multiple source data to enhance variation and weaken the blooming effect of DMSP-OLS light. Remote Sens. 2015, 7, 1422-1440.

51. Schölkopf, B.; Platt, J.C.; Shawe-Taylor, J.; Smola, A.J.; Williamson, R.C. Estimating the support of a high-dimensional distribution. Neural Comput. 2001, 13, 1443-1471.

52. Tax, D.M.J. One-Class Classification; Delft University of Technology: Delft, The Netherlands, 2001.

53. Sanchez-Hernandez, C.; Boyd, D.S.; Foody, G.M. One-class classification for mapping a specific land-cover class: SVDD classification of fenland. IEEE Trans. Geosci. Remote Sens. 2007, 45, 1061-1073.

54. Munoz-Mari, J.; Bruzzone, L.; Camps-Valls, G. A support vector domain description approach to supervised classification of remote sensing images. IEEE Trans. Geosci. Remote Sens. 2007, 45, 2683-2692.

55. Li, P.; Xu, H. Land-cover change detection using one-class support vector machine. Photogramm. Eng. Remote Sens. 2010, 76, 255-263.

56. Guo, Q.; Kelly, M.; Graham, C.H. Support vector machines for predicting distribution of sudden Oak death in California. Ecol. Model. 2005, 182, 75-90.

57. Liu, Z.; He, C.; Zhang, Q.; Huang, Q.; Yang, Y. Extracting the dynamics of urban expansion in China using DMSP-OLS nighttime light data from 1992 to 2008. Lands. Urban Plan. 2012, 106, $62-72$.

58. Deng, X.; Huang, J.; Rozelle, S.; Uchida, E. Growth, population and industrialization, and urban land expansion of China. J. Urban Econ. 2008, 63, 96-115.

59. National Bureau of Statistics of China. China Statistical Yearbook 1991; China Statistics Press: Beijing, China, 1991.

60. National Bureau of Statistics of China. China Statistical Yearbook 2013; China Statistics Press: Beijing, China, 2013.

61. Song, Y.; Ding, C. Urbanization in China: Critical Issues in An Era of Rapid Growth; Lincoln Institute of Land Policy: Cambridge, MA, USA, 2007.

62. Maimaitiming, A.; Xiaolei, Z.; Huhua, C. Urbanization in western China. Chin. J. Popul. Res. Environ. 2013, 11, 79-86.

63. Gu, J.; Li, X.; Huang, C.; Okin, G.S. A simplified data assimilation method for reconstructing time-series MODIS NDVI data. Adv. Space Res. 2009, 44, 501-509.

64. Huete, A.; Didan, K.; Miura, T.; Rodriguez, E.P.; Gao, X.; Ferreira, L.G. Overview of the radiometric and biophysical performance of the MODIS vegetation indices. Remote Sens. Environ. 2002, 83, 195-213.

65. Hulley, G.C.; Hook, S.J. Intercomparison of versions 4, 4.1 and 5 of the MODIS land surface temperature and emissivity products and validation with laboratory measurements of sand samples from the Namib desert, Namibia. Remote Sens. Environ. 2009, 113, 1313-1318.

66. Holben, B.N. Characteristics of maximum-value composite images from temporal AVHRR data. Int. J. Remote Sens. 1986, 7, 1417-1434.

67. Peng, S.; Piao, S.; Ciais, P.; Friedlingstein, P.; Ottle, C.; Breon, F.M.; Nan, H.; Zhou, L.; Myneni, R.B. Surface urban heat island across 419 global big cities. Environ. Sci. Technol. 2012, 46, 696-703. 
68. Zhao, L.; Lee, X.; Smith, R.B.; Oleson, K. Strong contributions of local background climate to urban heat islands. Nature 2014, 511, 216-219.

69. National Bureau of Statistics of China. China Statistical Yearbook 2007; China Statistics Press: Beijing, China, 2007.

70. Zhang, Q.; Schaaf, C.; Seto, K.C. The vegetation adjusted ntl urban index: A new approach to reduce saturation and increase variation in nighttime luminosity. Remote Sens. Environ. 2013, 129, 32-41.

71. Weng, Q. Thermal infrared remote sensing for urban climate and environmental studies: Methods, applications, and trends. ISPRS J. Photogramm. Remote Sens. 2009, 64, 335-344.

72. Gallo, K.P.; McNab, A.L.; Karl, T.R.; Brown, J.F.; Hood, J.J.; Tarpley, J.D. The use of a vegetation index for assessment of the urban heat island effect. Int. J. Remote Sens. 1993, 14, 2223-2230.

73. Landsberg, H.E. The Urban Climate; Academic Press: New York, NY, USA, 1981; Volume 28.

74. He, C.; Shi, P.; Li, J.; Chen, J.; Pan, Y.; Li, J.; Zhuo, L.; Ichinose, T. Restoring urbanization process in China in the 1990s by using non-radiance-calibrated DMSP/OLS nighttime light imagery and statistical data. Chin. Sci. Bull. 2006, 51, 1614-1620.

75. Huang, C.; Davis, L.; Townshend, J. An assessment of support vector machines for land cover classification. Int. J. Remote Sens. 2002, 23, 725-749.

76. Congalton, R.G.; Green, K. Assessing the accuracy of remotely sensed data: Principles and practices, second edition. CRC Press: Boca Raton, FL, USA, 2008.

77. Pontius, R.G., Jr.; Millones, M. Death to kappa: Birth of quantity disagreement and allocation disagreement for accuracy assessment. Int. J. Remote Sens. 2011, 32, 4407-4429.

78. Foody, G.M. Status of land cover classification accuracy assessment. Remote Sens. Environ. 2002, 80, 185-201.

79. Neubert, M.; Meinel, G. Evaluation of segmentation programs for high resolution remote sensing applications. In Proceedings of the International ISPRS Workshop "High Resolution Mapping from Space", Hannover, Germany, 6-8 October 2003.

(C) 2015 by the authors; licensee MDPI, Basel, Switzerland. This article is an open access article distributed under the terms and conditions of the Creative Commons Attribution license (http://creativecommons.org/licenses/by/4.0/). 